\title{
Fluoroquinolone and fortified antibiotics for treating bacterial corneal ulcers
}

\author{
Nibaran Gangopadhyay, Mark Daniell, LeAnn Weih, Hugh R Taylor
}

\begin{abstract}
Aim-To compare the clinical efficacy of commercially available fluoroquinolone drops with the use of combined fortified antibiotics (tobramycin 1.3\%-cefazolin 5\%) in treatment of bacterial corneal ulcer. Methods-The medical records of 140 patients with a diagnosis of bacterial corneal ulcer who were admitted to the Royal Victorian Eye and Ear Hospital, Melbourne, Australia between January 1993 and December 1997 were reviewed retrospectively. Final outcome and results of 138 ulcer episodes were compared between those treated initially with fluoroquinolone and those who received fortified antibiotics. Two patients had been treated with chloramphenicol.

Results-No significant treatment difference was found between fluoroquinolone and fortified therapy in terms of final visual outcome. However, serious complications such as corneal perforation, evisceration, or enucleation of the affected eye were more common with fluoroquinolone therapy $(16.7 \%)$ compared with the fortified therapy $(2.4 \%, p=0.02)$. The duration of intensive therapy was less with fluoroquinolone especially in those over 60 years of age ( 4 days $v 6$ days, $p=0.01$ ). Hospital stay was also less in the fluoroquinolone group compared with the fortified group for all patients and was significantly less with fluoroquinolone treatment ( 7 days $v$ 10 days, $p=0.02$ ) in patients in the age group over 60 years old.
\end{abstract}

Conclusions-Monotherapy with fluoroquinolone eye drops for the treatment of bacterial corneal ulcers led to shorter duration of intensive therapy and shorter hospital stay compared with combined fortified therapy (tobramycin-cefazolin). This finding may have resulted from quicker clinical response of healing as a result of less toxicity found in the patients treated with fluoroquinolone. However, as some serious complications were encountered more commonly in the fluoroquinolone group, caution should be exercised in using fluoroquinolones in large, deep ulcers in the elderly.

(Br F Ophthalmol 2000;84:378-384)

The accepted treatment for severe bacterial corneal ulcers includes frequent administration of fortified topical ocular antibacterial agents (usually a cephalosporin and an aminoglycoside) used together to cover the maximum spectrum of bacteria. Treatment is modified according to host clinical response and laboratory susceptibility data concerning the organism(s) along with decisions regarding adjunctive therapy. ${ }^{1-3}$

There are a few shortcomings of this regimen. Frequent dosing of multiple antibiotics simultaneously may result in increased toxicity and damage to the ocular surface epithelium. ${ }^{2}$ The increased tonicity of fortified drops induces reflex tearing which, in addition to the dilution that occurs secondary to osmosis, may actually decrease tissue penetration. The fortified preparations have a variable and shorter shelf life. Moreover, each requires special mixing by a pharmacist which adds to cost and increases risk of contamination. ${ }^{4-7}$

The introduction of a commercially available, non-fortified broad spectrum topical antibiotic with the ability to achieve concentrations greater than the minimum inhibitory concentration for most bacteria was desirable. Ofloxacin and ciprofloxacin are fluoroquinolone antibiotics with broad spectrum antibacterial activity against most aerobic Gram positive and Gram negative bacteria, mycobacteria, mycoplasma, and chlamydiae including methicillin resistant Staphylococcus aureus and Pseudomonas aeruginosa. ${ }^{9}$ In recent clinical trials, their topical application has been shown to be effective in the treatment of bacterial keratitis caused by the most commonly encountered organisms. ${ }^{10-15}$ A single agent that is commercially available has other advantages such as lowered dispensing cost, elimination of potential contamination, stable $\mathrm{pH}$, and longer shelf life. ${ }^{16}$

For a number of years, the corneal unit of the Royal Victorian Eye and Ear Hospital has used a standard treatment protocol for the treatment of corneal ulcers. Before April 1995 the protocol was to initially treat all patients with 1 hourly fortified topical antibiotics (cefazolin 5\% and tobramycin 1.3\%) combined together after the collection of corneal scraping for microbiology and culture. In April 1995 the protocol changed to use 1 hourly fluoroquinolone eye drops.

\section{Material and methods}

The medical records of all patients diagnosed with bacterial corneal ulcer who were admitted (first admissions only during this period) to the Royal Victorian Eye and Ear Hospital in Melbourne, between January 1993 and December 1997, were selected for retrospective review.

Diagnosed fungal or viral ulcers were not included in this study. The diagnosis of bacterial corneal ulcer was based on supportive clinical findings confirmed by microbiology (stain and culture). 
The clinical records of 140 patients with a history of bacterial ulcer episodes were reviewed with attention to the nature of the organism and its sensitivity, to identify the presence of associated ocular and systemic conditions, and concurrent factors for ulcer occurrence, use of topical steroid, healing, visual outcome, complications, duration of intensive therapy, and total hospital stay. These factors and other pertinent features were compared in the two groups of patients $(n=138)$, one treated with fluoroquinolone only and the other with combined fortified therapy. Two patients had been treated with chloramphenicol alone and they are not included in the statistical analysis.

STATISTICAL METHODS

Data for this study were double entered and verified using Paradox (Borland). Statistical analysis was performed using SPSS, version 8 . In the analyses, both days of hospital stay and of intensive therapy were transformed by the natural logarithm of days in order to approximate a normal distribution. To identify potential confounding factors and to evaluate univariate associations with duration of hospital stay or intensive therapy, $\chi^{2}$ tests are used to evaluate differences in proportions and unpaired $t$ tests are used to evaluate differences in means between the fluoroquinolone and fortified therapy groups. Analysis of variance is used to evaluate differences in duration of hospital stay or intensive therapy, controlling for confounding variables. The natural log of hospital days and intensive therapy days was used

Table 1 Demographic characteristics of 140 patients with bacterial keratitis

\begin{tabular}{llll}
\hline Factors & $\begin{array}{l}\text { Fluoroquinolone } \\
(n=54)(38.6 \%)\end{array}$ & $\begin{array}{l}\text { Fortified-Combined } \\
(n=84)(60 \%)\end{array}$ & $\begin{array}{l}\text { Total numbers } \\
(n=140)(100 \%)\end{array}$ \\
\hline Sex: $\mathrm{M} / \mathrm{F}$ & $32 / 22(59.3 / 40.7)$ & $51 / 33(60.7 / 39.3)$ & $83 / 55(59.3 / 40.7 \%)$ \\
$\begin{array}{l}\text { Mean (SD) age (years) } \\
\text { Ulcer location: }\end{array}$ & $72.2(20.4)$ & $61.2(23.7)$ & $65.5(23.0)$ \\
$\quad \begin{array}{l}\text { Central } \\
\quad \begin{array}{l}\text { Peripheral } \\
\text { Limbal }\end{array}\end{array}$ & $39(72.2 \%)$ & $50(59.5 \%)$ & $89(64.5 \%)$ \\
Ulcer depth & $11(20.4 \%)$ & $32(38.1 \%)$ & $43(31.2 \%)$ \\
$\quad$ Superficial <1/3 & $4(7.4 \%)$ & $2(2.4 \%)$ & $6(4.3 \%)$ \\
$\quad$ Deep >1/3 & $22(41.5 \%)$ & $43(51.8 \%)$ & $65(47.8 \%)$ \\
Mean ulcer size $\left(\mathrm{mm}^{2}\right)$ & $31(58.5 \%)$ & $40(48.2 \%)$ & $71(52.2 \%)$ \\
Hypopyon & $11.0(64.8 \%)$ & 7.9 & 9.2 \\
Vascularisation & $35(50 \%)$ & $77(54 \%)$ \\
Stromal thinning & $21(38.9 \%)$ & $23(27.4 \%)$ & $44(31.4 \%)$ \\
\hline
\end{tabular}

Table 2 Prestudy pathology and therapy of 140 ulcer events

\begin{tabular}{lccc}
\hline Pathology & Fluoroquinolone & Fortified-Combined & Total numbers \\
\hline Corneal conditions: & & & \\
$\quad$ Scar & $35(64.8 \%)$ & $45(53.5 \%)$ & $70(48.6 \%)$ \\
HSV stromal keratitis & $13(24.0 \%)$ & $13(14.0 \%)$ & $26(18.2 \%)$ \\
Trophic cornea & $7(13.0 \%)$ & $9(9.6 \%)$ & $16(11.6 \%)$ \\
$\quad$ Oedema & & & \\
Other conditions: & $4(7.4 \%)$ & $4(4.8 \%)$ & $8(5.6 \%)$ \\
$\quad$ Pterygium & $7(13.0 \%)$ & $11(11.7 \%)$ & $18(13.0 \%)$ \\
Spheroidal degeneration & $4(7.4 \%)$ & $8(9.5 \%)$ & $12(8.7 \%)$ \\
$\quad$ Inflammation & $12(22.3 \%)$ & $13(15.5 \%)$ & $25(18.1 \%)$ \\
Associated lid conditions: & $9(16.7 \%)$ & $8(9.5 \%)$ & $17(12.3 \%)$ \\
$\quad$ Lagophthalmos & & & \\
Other conditions: & $7(13.0 \%)$ & $18(13.0 \%)$ & $25(18.1 \%)$ \\
$\quad$ Trauma & $26(48.0 \%)$ & $27(32.0 \%)$ & $53(38.4 \%)$ \\
Past surgery & $7(13.0 \%)$ & $1(1.4 \%)$ & $8(5.8)$ \\
Dry eye & $12(22.2 \%)$ & $18(21.4 \%)$ & $30(21.7 \%)$ \\
Glaucoma & $5(9.2 \%)$ & $7(8.3 \%)$ & $12(8.7 \%)$ \\
Viral keratitis +Rx & $12(22.2 \%)$ & $24(26.0 \%)$ & $36(26.0 \%)$ \\
Antiglaucoma Rx & $5(9.2 \%)$ & $14(16.6 \%)$ & $19(14.4 \%)$ \\
$\quad$ Contact lens & & & \\
\hline
\end{tabular}

in the analyses of variance. Differences in length of hospital stay, duration of intensive therapy, time to resolution of infiltration, and time to complete healing of epithelial defect between the two therapy groups was also evaluated using Kaplan-Meier life table analysis. Multivariable logistic regression analysis was used to evaluate risk of complications by treatment group controlling for confounding variables. Forward conditional selection was used to select the final model. In addition to analysis of the study sample as a whole, the treatments and outcomes were compared between the ofloxacin and ciprofloxacin cases as a subgroup of monotherapy with fluoroquinolone.

\section{Results}

PATIENT DEMOGRAPHICS

A total of 140 patient charts were reviewed with 140 ulcer events. Of the total 140 ulcer events which were evaluated for protocol, 54 $(38.6 \%)$ ulcers were treated with fluoroquinolone, $84(60 \%)$ ulcers were treated with fortified tobramycin-cefazolin, and two $(1.4 \%)$ patients were treated with chloramphenicol. These two patients are described.

There were no significant differences between the treatment groups for any of the demographic characteristics except for age of presentation (Table 1). The patients treated with fluoroquinolone were older than those in the fortified group.

There were no statistically significant differences between the treatment groups for any of the other baseline characteristics including ulcer size, depth, or location, for presence of vessels, stromal thinning, or hypopyon. In the fluoroquinolone group the mean ulcer size was $11 \mathrm{~mm}^{2}$ and in the fortified combined group it was $7.9 \mathrm{~mm}^{2}$ and the mean ulcer size was 9.2 $\mathrm{mm}^{2}$. Information on depth of two ulcers was missing from the records.

No significant differences between the two treatment groups were found in terms of presence of lid pathology, corneal pathology, or other concurrent eye conditions (Table 2).

The presence of other associated eye conditions with potential to act as predisposing factors for the ulcer such as trauma, past surgical intervention, dry eye, glaucoma, and contact lens wear was evaluated and no significant differences in distribution between the two treatment groups were found.

\section{CONTACT LENS WEAR}

Nineteen $(14.4 \%)$ ulcer cases were associated with contact lens use, of which five $(9.2 \%)$ were in the fluoroquinolone group and 14 $(16.6 \%)$ were in the fortified combined group. (Table 2). Fifteen patients used contact lens for refractive reasons and 13 were soft contact lenses. Four were bandage contact lenses used for corneal conditions and three of these were in the fluoroquinolone group. More patients in the fortified group had a history of contact lens wear but this was not significant. There was no specific preponderance of particular bacteria noted in the contact lens related ulcers. 
PRE-EXISTING SYSTEMIC DISEASES

Patients in the fluoroquinolone group were more likely to have systemic diseases (rheumatoid arthritis, diabetes, hypertension, asthma, gout, peptic ulcer, ischaemic heart disease (IHD), or others) compared with those in the fortified group (Table 3). Of 140 ulcer episodes, $71(50.7 \%)$ were associated with one or more of those systemic diseases and 37 $(68.5 \%)$ of them were in the fluoroquinolone group.

Patients taking fluoroquinolone therapy were more likely to be concurrently using systemic immunosuppressive drugs than the patients taking fortified therapy. Seventeen $(12 \%)$ ulcer episodes were associated with concurrent use of topical steroids, six (11\%) of which were in the fluoroquinolone group, and $11(13 \%)$ in the fortified group. Seven (5\%) ulcer episodes were found to be associated with concurrent systemic steroid use and six of them were in the fluoroquinolone group. Of these six patients three were also taking antimetabolites for rheumatoid arthritis.

VISUAL ACUITY ON PRESENTATION

There was a significant imbalance in the distribution of visual acuity on presentation between the two therapy groups, in the extremes of visual acuity range (Fisher's exact $\mathrm{p}<0.001$ ) (Table 4).

Table 3 Pre-existing systemic diseases in 140 ulcer episodes

\begin{tabular}{lccl}
\hline Systemic diseases & Fluoroquinolone & Fortified-Combined & $\begin{array}{l}\text { Total }(n=71) \\
(51.3 \%)\end{array}$ \\
\hline $\begin{array}{l}\text { Diabetes } \\
\text { Hypertension }\end{array}$ & $9(16.7 \%)$ & $9(10.7 \%)$ & $18(13.0 \%)$ \\
$\begin{array}{l}\text { Rheumatoid arthritis } \\
\begin{array}{l}\text { Others (asthma, gout, } \\
\text { peptic ulcer, IHD) }\end{array}\end{array}$ & $10(18.5 \%)$ & $11(13.1 \%)$ & $21(15.2 \%)$ \\
\hline
\end{tabular}

Table 4 Visual acuity on presentation of 140 ulcer episodes

\begin{tabular}{lccl}
\hline Visual acuity & Fluoroquinolone & Fortified-Combined & Total \\
\hline $6 / 5$ to $6 / 9$ & $1(1.8 \%)$ & $14(16.7 \%)$ & $15(10.8 \%)$ \\
$6 / 12$ to $6 / 24$ & $7(13.0 \%)$ & $20(23.8 \%)$ & $27(19.3 \%)$ \\
$6 / 36$ to $6 / 60$ & $6(11.1 \%)$ & $8(9.5 \%)$ & $14(10.0 \%)$ \\
$3 / 60$ to $\mathrm{HM}, \mathrm{PL}^{\star}$ & $37(68.5 \%)$ & $28(33.3 \%)$ & $67(47.8 \%)$ \\
NPL & $3(5.6 \%)$ & $14(16.7 \%)$ & $17(12.1 \%)$ \\
\hline
\end{tabular}

^Two patients treated with chloramphenicol had vision of 3/36 and HM.

Table 5 Microbiological analysis of 122 isolates of 100 culture positive ulcers

\begin{tabular}{ll}
\hline Gram positive bacteria & Number \\
\hline Staphylococcus epidermidis and coagulase negative & 32 \\
Staphylococcus aureus & 27 \\
Streptococcus pneumoniae & 9 \\
Other Streptococcus sp & 7 \\
Bacillus cereus & 1 \\
Corynebacterium sp & 13 \\
Propionibacterium acnes & 6 \\
Total No (\%) of Gram positive bacteria & $95(79.0 \%)$ \\
\hline Gram negative bacteria & Number \\
\hline Pseudomonas sp & 9 \\
Enterobactor cloacae & 1 \\
Escherichia coli & 1 \\
Proteus mirabilis & 3 \\
Haemophilus influenzae & 1 \\
Moraxella sp & 9 \\
Serratia marcescens & 1 \\
Total No (\%) of Gram negative bacteria & $25(21.0 \%)$ \\
Total No of isolates & $122(100 \%)$ \\
\hline
\end{tabular}

MICROBIOLOGICAL ANALYSIS

Laboratory data in all 140 study ulcers were available. Of 140 Gram stain specimens, 56 (40\%) specimens showed organism on Gram stain. Of these, 35 (62.5\%) Gram positive cocci, 12 (21.4\%) were Gram positive bacilli (four of them mixed with Gram positive cocci and one with Gram negative cocci), eight $(14.3 \%)$ were Gram negative bacilli, and one $(1.8 \%)$ was Gram negative cocci (mixed with Gram positive bacilli). Five specimens showed mixed organisms. There was no difference in yields in Gram stain between the therapy groups.

Of 140 culture results available for analysis, $100(71.4 \%)$ cases were culture positive yielding 120 isolates. Sixteen (16\%) were a combination of two or more isolates; four cultures were a mix of three isolates (Table 5).

Of 16 cases of polyisolates, nine had Staphylococcus $\mathrm{sp}$ mixed with another organism (three with Streptococcus, three with Corynebacterium, two with Proteus, and one with Moraxella), three were a combination of two different Staphylococcus sp, and four had Staphylococcus sp mixed with two other isolates (three with Moraxella and Corynebacterium and one with Moraxella and Streptococcus). The distribution of type of bacterial organisms was similar in each therapy group and no significant difference in percentage of isolates between therapy groups including the polybacterial infections was observed. One of the patients treated with chloramphenicol grew Serratia marcescens in culture and the other one grew mixed $S$ epidermidis with a coagulase negative Staphylococcus sp.

\section{OFLOXACIN $V$ CIPROFLOXACIN THERAPY}

Fifteen patients in the fluoroquinolone group were treated with ciprofloxacin and 39 were treated with ofloxacin. No difference in demographic features (mean age, sex, ulcer type, location and depth, hypopyon, thinning, lid pathology, or presence of other eye conditions) was noted between the ciprofloxacin and ofloxacin group. No difference was noted for use of concurrent steroid therapy, immunosuppression, or use of topical steroid during healing between these two therapy groups. Microbiological data in the ciprofloxacin group were found to have similar characteristics as in the ofloxacin group. Final visual outcome, types, and frequency of complications encountered and total duration of intensive therapy and hospital stay between the ciprofloxacin and ofloxacin groups were without any significant differences. Only one patient developed a corneal precipitate at the ulcer site after treatment and this was with ciprofloxacin.

\section{TREATMENT MODIFICATIONS}

The treatment protocol for 16 (11.4\%) patients out of 140 was modified either because of a delay in expected clinical response or concern about antibiotic resistance. Ten (12\%) were in the fortified group and six (11\%) received fluoroquinolones (Table 6). Only two patients had a confirmed resistant organism to therapy (cefazolin in each case). 
Table 6 Reasons for treatment modifications of 16 patients

\begin{tabular}{|c|c|c|c|c|c|c|}
\hline Patient No & Therapy & Organism & Resistance & Reasons & Modification & Outcome \\
\hline 1 & Fluoroquinolone & $\begin{array}{l}S \text { aureus and } \\
\quad \text { (S aureus from leg ulcer) }\end{array}$ & $\begin{array}{l}\text { Cefazolin (resist } \\
\text { to all except } \\
\text { vanco) }\end{array}$ & $\begin{array}{l}\text { Concern about } \\
\text { sensitivity (CAS) }\end{array}$ & $\begin{array}{l}\text { Topical cefazolin, systemic } \\
\text { vancomycin, cefrataxone }\end{array}$ & PED conj flap \\
\hline 2 & Fluoroquinolone & $S$ pneumoniae & - & CAS & $\begin{array}{l}\text { Topical cefazolin, penicillin, } \\
\text { systemic penicillin }\end{array}$ & Healing at last exam \\
\hline 3 & Fluoroquinolone & $S$ aureus & - & CAS & Topical vancomycin & Healed \\
\hline 4 & Fluoroquinolone & Pacnes & - & CAS & Topical tobramycin & PED SBCL applied \\
\hline 5 & Fluoroquinolone & S pneumoniae & - & CAS & Topical cefazolin & Healing at last exam \\
\hline 6 & Fluoroquinolone & Moraxella & - & $\begin{array}{l}\text { Deterioration } \\
\text { Hypopyon developed }\end{array}$ & Topical cefazoloin & Healed \\
\hline 7 & Fortified & $\begin{array}{l}\text { S pneumoniae } \\
\text { S aureus }\end{array}$ & - & CAS & $\begin{array}{l}\text { Topical vancomycin, systemic } \\
\text { cefazolin and ciprofloxacin }\end{array}$ & PED tarsorrhaphy \\
\hline 8 & Fortified & $S$ haemolyticus & & CAS & $\begin{array}{l}\text { Subconjunctival gentamicin, } \\
\text { systemic ciprofloxacin and } \\
\text { penicillin }\end{array}$ & $\begin{array}{l}\text { Eventual } \\
\text { enucleation }\end{array}$ \\
\hline 9 & Fortified & $S$ sanguis & & CAS & To pical vancomycin & Healed \\
\hline 10 & Fortified & Enterobactor cloacae & Cefazolin & Resistant organism & Topical ciprofloxacin & Healed \\
\hline 11 & Fortified & Moraxella $\mathrm{sp}$ & Cefazolin & Resistant organism & $\begin{array}{l}\text { Systemic ciprofloxacin and } \\
\text { gentamicin, topical antiviral }\end{array}$ & PED conj flap \\
\hline 12 & Fortified & $P$ aeruginosa & & CAS & $\begin{array}{l}\text { Systemic cefazolin, gentamicin, } \\
\text { and antiviral }\end{array}$ & Healing \\
\hline 13 & Fortified & $P$ aeruginosa & Chloramph enicol & & Systemic ticarcillin & Healed \\
\hline 14 & Fortified & P aeroginosa & Chloramph enicol & & Systemic ticarcillin & PED conj flap \\
\hline 15 & Fortified & $\begin{array}{l}\text { Alpha haemolytic strep, } S \\
\quad \text { epidermidis, and coag -ve }\end{array}$ & & Deterioration & Systemic cefazolin and tobramycin & PK \\
\hline 16 & Fortified & $S$ aureus & & History of $\mathrm{HZO}$ & $\begin{array}{l}\text { Systemic ciprofloxacin and } \\
\text { topical antiviral }\end{array}$ & Healed \\
\hline
\end{tabular}

Two patients were treated with topical chloramphenicol. One woman aged 84 had a corneal ulcer secondary to dry eye and lagophthalmos that was thought to be sterile. She improved with topical intensive chloramphenicol eye drops and lubricants. The other, a woman aged 90 , had a pre-existing epithelial defect on a scarred cornea which had developed a secondary infection with Serratia marcescens that responded well.

\section{STEROID THERAPY}

Topical steroid was used as an adjunct therapy to facilitate the healing by decreasing inflammation once the infection was clinically controlled and appropriate antibiotic therapy was supported by culture and sensitivity reports. Usually, topical steroid was started when intensive antibiotic therapy was tapered. No steroids were used in the two patients treated with topical chloramphenicol. Four topical steroid preparations were used predominantly: Prednefrin forte (prednisolone acetate $1 \%$ ), Flarex (fluorometholone acetate $0.1 \%$ ), Predsol (prednisolone sodium phosphate $(0.5 \%)$, or Predsol minims (no preservative). FML (fluorometholone $0.1 \%$ ) or Maxidex (dexamethasone $0.5 \%$ ) were used infrequently.

Steroid eye drops were used in $88(62.7 \%)$ cases. Of the 36 cases in the fluoroquinolone group, $24(66.6 \%)$ ulcers received Flarex drops and $12(33.4 \%)$ received other steroid drops. In the fortified group, 22 of 52 cases $(42.3 \%)$ received Prednefrin forte drops. Flarex was used more in the fluoroquinolone group $\left(\chi^{2}=17.6, \mathrm{p}<0.001\right)$.

Table 7 Multivariate analysis of serious complications controlling for age, systemic disease, and immunosuppression

\begin{tabular}{llll}
\hline & Fortified & Fluoroquinolone & p Value \\
\hline Enucleated/eviscerated & $2(2.4 \%)$ & $4(7.4 \%)$ & 0.2 \\
Perforated & $0(0 \%)$ & $5(9.3 \%)$ & 0.9 \\
Serious complications & $2(2.4 \%)$ & $9(16.7 \%)$ & 0.02 \\
PED & $5(5.9 \%)$ & $0(0 \%)$ & 0.1 \\
Any complications & $7(8.3 \%)$ & $9(16.7 \%)$ & 0.1 \\
\hline
\end{tabular}

COMPLICATIONS

Nine $(16.7 \%)$ of the patients treated with fluoroquinolone had serious complications (perforation or enucleation and evisceration) compared with two $(2.4 \%)$ in the fortified group (Fisher's exact, $\mathrm{p}=0.004$ ) (Table 7). Perforation was noted in five cases, all in the fluoroquinolone group. Six patients' eyes were enucleated or eviscerated; four of them were in the fluoroquinolone group and two of them were in the fortified group. Of five ulcers with perforation, three had to be treated with glue and bandage contact lens to provide tectonic support and two of them were treated with emergency grafting. In total, four ulcers underwent urgent keratoplasty. The other two were large and deep ulcers in the combined fortified therapy group.

Serious complications were unrelated to the presence of any systemic disease, steroid medication, or immune supplementation in either univariate or multivariate models. Controlling for age and visual acuity at admission, the fluoroquinolone therapy group had an 8.9-fold (95\% CI 1.5, 51.7) increased risk of serious complication. Each decrement of visual acuity on admission was associated with a marginally significant increased risk of serious complication. Controlling for systemic disease and steroid medication in addition to age and visual acuity, the fluoroquinolone therapy group remained at increased risk of serious complication. Subanalyses of the patients who were over 60 years of age yielded similar results.

\section{LENGTH OF HOSPITAL STAY AND INTENSIVE}

THERAPY

For the people above the age of 60 years controlling for age, systemic diseases, and immunosuppression (topical and systemic steroid or antimetabolites), there was a significant reduction in the duration of intensive therapy in the fluoroquinolone group, 4 days, when compared with those treated with fortified drops, 6 days $(p<0.001)$. Intensive 

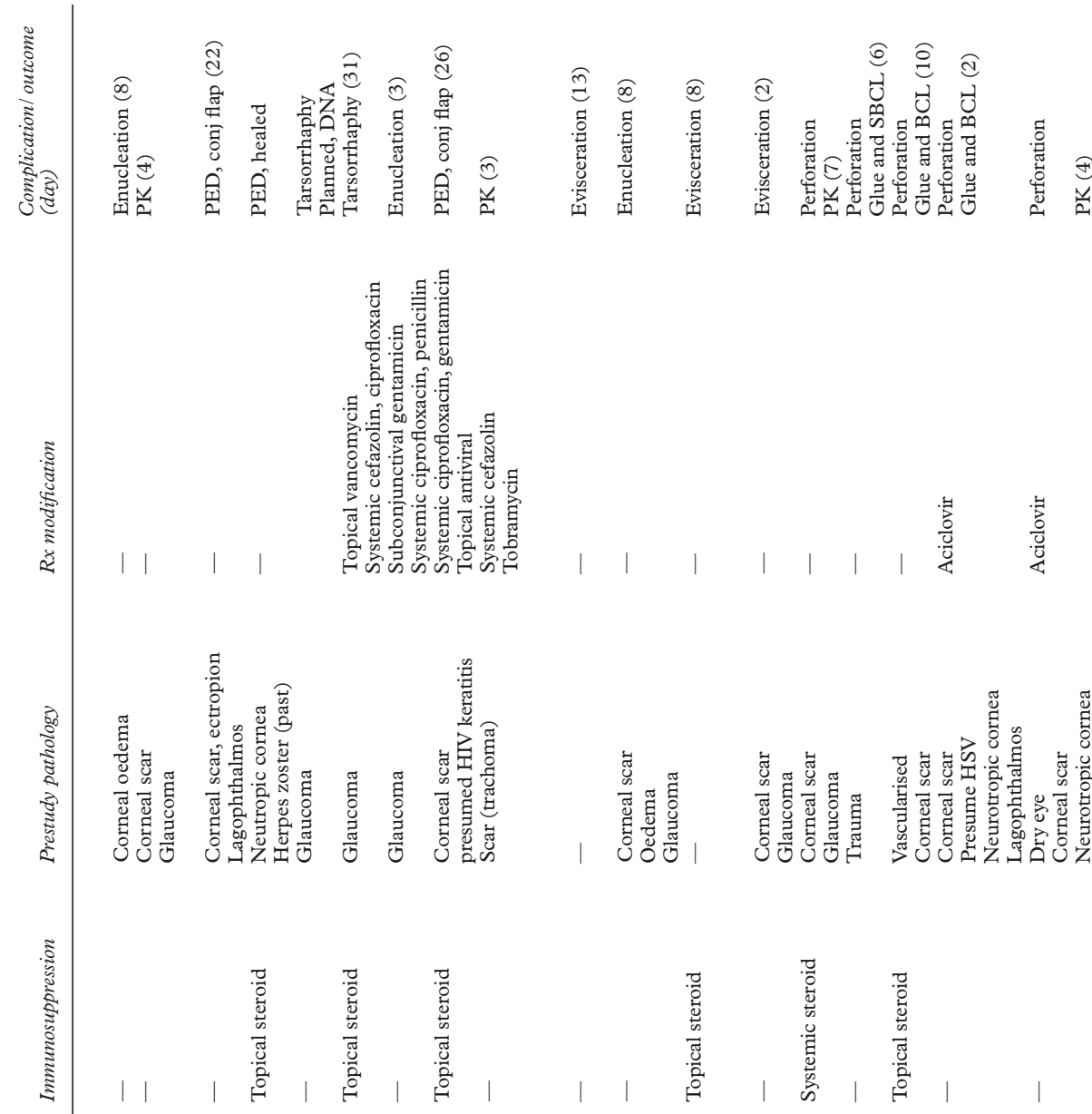

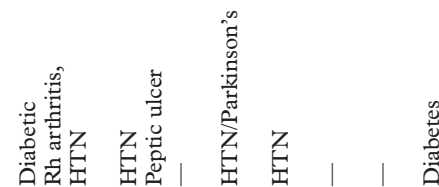

星自

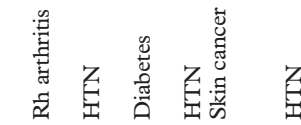

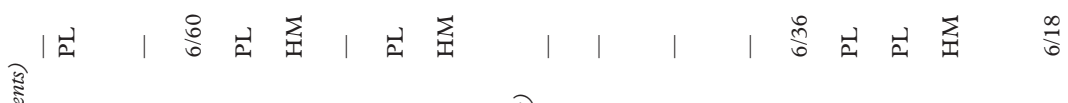

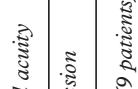

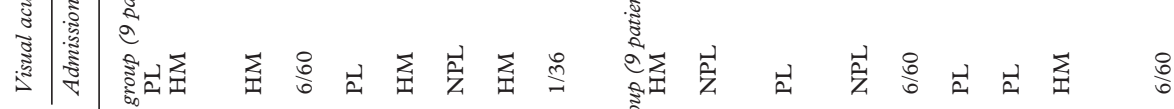


therapy was defined as application of drops from every hour to every $2-3$ hours.

For the people older than 60 years of age the median hospital stay was longer in the fortified group, 10 days, compared with 7 days in the fluoroquinolone group $(\mathrm{p}=0.01)$.

VISUAL OUTCOME AT LAST FOLLOW UP VISIT Of 140 patients, data on the visual acuity of 77 patients (whose ulcers were documented as completely healed, was analysed. All patients (five in the fluoroquinolone group and 21 in the fortified group) whose visual acuity on presentation was $6 / 18$ or better did not show any change in their visual acuity after healing at the last follow up. Of 29 patients in the fortified group who had a visual acuity less than $6 / 18$ on presentation, nine (31\%) improved to $6 / 18$ whereas only one $(4.5 \%)$ patient out of 22 in the fluoroquinolone group with a visual acuity less than $6 / 18$ improved to $6 / 18$. Thus, visual outcome in the patients with already poor vision was worse in the fluoroquinolone group $\left(\mathrm{p}=0.02 \chi^{2} \mathrm{df}=5.6\right)($ Table 8$)$.

\section{Discussion}

Clinical studies have shown that treatment outcomes with fluoroquinolone monotherapy (either ciprofloxacin or ofloxacin) compare favourably with conventional combined therapy of fortified antibiotics. ${ }^{10-15}$ In our study we not only found that the response to fluoroquinolone was comparable with that of fortified therapy, but also found a shorter duration of intensive therapy ( 4 days $v 6$ days), and an associated shortened hospital stay (7 days $v 10$ days) in the patients treated with fluoroquinolone drops. The reduced duration of intensive therapy along with shortened hospital stay are good indicators of earlier and better clinical response in the patients treated with fluoroquinolone drops. Our study, being retrospective in nature, lacks the detailed documentation of objective evidence of toxicity from therapy but one could assume that patients with less toxicity and an earlier healing had intensive therapy tapered more quickly and so a shorter hospital stay. There was no other policy change over this period in relation to duration of hospitalisation.

Leibowitz, in a multicentre study of patients with culture positive infectious keratitis, observed $92 \%$ success with ciprofloxacin. ${ }^{10}$ Similarly, Wilhelmus et al found that clinical success occurred in $93 \%$ of the patients treated with ciprofloxacin ointment used for a shorter mean duration than conventional agents. ${ }^{11}$ In this prospective study more patients in the non-enrolled group treated with conventional therapy experienced failure than in the ciprofloxacin group. The recent clinical trial by the ofloxacin study group showed no difference in treatment success between the two treatments with $62.1 \%$ of the ofloxacin group and $67.9 \%$ of the conventional treatment group being cured within 14 days. $^{15}$

Some of these studies have shown a significant reduction in the incidence of ocular discomfort or objective evidence of reduced toxicity associated with fluoroquinolone therapy. ${ }^{13-15}$ O'Brien et $a l^{13}$ found eight of nine patients with severe ocular side effects were in the fortified antibiotic group. The ofloxacin study group noticed five times more toxicity encountered with the conventional therapy and significantly less objective evidence of toxicity associated with ofloxacin therapy. ${ }^{15}$ The most frequently reported adverse event with ciprofloxacin treatment was development of transient white precipitate in $13-42 \%$ cases. $^{11}$ Unlike ciprofloxacin, ofloxacin has not been reported to cause corneal precipitate during intensive use.

However, there are fewer studies regarding any other benefit of fluoroquinolones in the treatment of bacterial keratitis. In one large study of ciprofloxacin monotherapy for bacterial keratitis, Wilhelmus et $a l^{11}$ found that the mean duration of treatment with ciprofloxacin ointment (18 (SD 10) days) was significantly shorter than that with conventional therapy (24 (16) days).

In this study, fewer patients with poor vision on presentation showed an improvement in final visual acuity in the fluoroquinolone group than in the fortified group. The lack of improvement in vision in the fluoroquinolone group is undoubtedly influenced by the higher rate of adverse outcomes, but is a worrying trend. Earlier studies by O'Brien et $a l^{13}$ and Hyndiuk et $a l,{ }^{14}$ or by the ofloxacin study group ${ }^{15}$ have not addressed the important issue of visual outcome.

The choice of initial therapy for suspected microbial keratitis should be governed or guided by contemporary epidemiological findings. The microbiological findings in the current study are similar with other studies. ${ }^{111314}$ However, the ofloxacin study group ${ }^{15}$ had a higher incidence of Pseudomonas ulcers, $26.5 \%$, compared with the $6.4 \%$ in our study. Also there was a greater incidence, $39 \%$, of contact lens wear ${ }^{14}{ }^{15}$ compared with $13.5 \%$ of contact lens wear in our study. Our study was based on patients who required admission, who are presumably the most severe cases. This factor may have contributed to some of the differences with other studies.

We found that all isolates of Streptococcus sp were sensitive to fluoroquinolone, tobramycin, and cefazolin. In fact, we did not encounter any isolate that was resistant to fluoroquinolone.

However, the continued use of an antibiotic raises the issue of emerging resistance. ${ }^{17-19}$ The ofloxacin study group ${ }^{15}$ had $6.1 \%$ resistant organisms to ofloxacin and O'Brien et al ${ }^{13}$ reported $2 \%$ resistant. Recently, Kunimoto et $a l^{19}$ have reported $30.7 \%$ (478 out of 1558 ) of corneal isolates in India were resistant to ciprofloxacin in vitro. It should be noted that drug levels attainable in the cornea are much higher than those in serum, especially with repeated dosing, and so relative resistance of an organism in vitro may not result in treatment failure.

In our study, a significant unexpected finding was the incidence of serious complications in the fluoroquinolone group, most notably corneal perforation. Patients in this group were older on presentation and had more 
associated systemic diseases such as rheumatoid arthritis. The ulcers that perforated all had severe ocular problems in addition to microbial keratitis. All these eyes had very poor visual potential as a result of other coexisting ocular pathologies. All of these cases involved perforated in the first few days of treatment and may just reflect the severity of the presenting pathology. This, in fact, is a significant outcome in multivariable models (see Table 7 ).

An alternative explanation is that the fluoroquinolone may cause alteration in the tectonic strength of the cornea. There is emerging evidence of a rate of spontaneous Achilles tendon rupture in patients taking systemic fluoroquinolones. This is thought to be related to focal necrosis within the tendon. Increasing age, systemic steroids, and renal failure are risk factors. ${ }^{20}$

In our patients perforation was related to large deep ulcers in elderly patients. It is conceivable that changes to the stromal collagen as a result of fluoroquinolone treatment could have reduced the tectonic strength of the cornea and increased the risk of perforation. On the basis of these results, use of combined fortified antibiotics rather than fluoroquinolones could be considered in cases at higher risk of perforation.

\section{Conclusion}

The acute management of a bacterial corneal ulcer requires urgent access to appropriate therapy. In addition, the cost and toxicity of therapy must be considered. Clearly, in terms of accessibility, cost, and toxicity, the advantage belongs to the fluoroquinolone. Moreover fluoroquinolone drops have a stable $\mathrm{pH}$ and shelf life as they are readily available ${ }^{716}$

The use of fluoroquinolones as monotherapy for bacterial keratitis has proved as effective as combined fortified antibiotics and may have some advantages with decreased toxicity and duration of treatment. There was a similar rate of adverse events but with a different distribution, including a higher rate of corneal perforation. Caution should be exercised in using fluoroquinolones in large, deep ulcers in the elderly.
We thank Mrs Cara Jin, Dr Cathy McCarty, and Dr B N Mukesh.

1 Baum JL, Barza M, Weinstein L. Preferred routes of antibiotic administration and treatment of bacterial ulcers of the cornea. Int Ophthalmol Clin 1973;13:31-7.

2 Baum JL, Jones DB. Initial therapy of suspected microbial corneal ulcers. I: Broad antibiotic therapy based on prevaence of organisms. II: Specific antibiotic therapy based on corneal smears. Surv Ophthalmol 1979;24:97-116.

3 Hyndiuk RA, Cokington CD. Bacterial keratitis. In: Tabara $\mathrm{KF}$, Hyndiuk RA, eds. Infections of the eye. 2nd ed. Boston: Little Brown, 1996:chapter 22:323-47.

4 Glasser DB, Gardner S, Ellis JG, et al. Loading doses and extended dosing intervals in topical gentamicin therapy. Am 7 Ophthalmol 1985;99:329-32.

5 Baum JL. Treatment of bacterial ulcers of the cornea in the rabbit: a comparison of administration by eye drops and subconjunctival injections. Trans Am Ophthalmol Soc 1982; subconjunctivis

6 Ronaldo M, Brezzo V, Campagna P, et al. Toxic effects of antimicrobials on the ocular surface of healthy volunteers. Chibret Int F Ophthalmol 1991;8:46-50.

7 Bowe BE, Snyder JW, Eiferman RA. An in vitro study of the potency and stability of fortified ophthalmic antibiotic preparation. Am f Ophthalmol 1991;111:686-9.

8 Wolfson JS, Hooper DC, eds. Quinolone antimicrobial agents. 2nd ed. Wash

$$
\text { ogy, } 1993
$$

9 Pendltton KM, Hobden JA, Hill JM, et al. Antimicrobial activity of ciprofloxacin against organisms isolated from patients bacterial keratitis. Invest Ophthalmol Vis Sci 1991;32:1171-6.

10 Leibowitz HM. Clinical evaluation of ciprofloxacin $0.3 \%$ ophthalmic solution for treatment of bacterial keratitis. $\mathrm{Am}$ f Ophthalmol 1991;112:34S-47S

11 Wilhelmus KR, Hyndiuk RA, Caldwell DR, et al. 0.3\% Ciprofloxacin ophthalmic ointment in the treatment of bacterial keratitis. Arch Ophthalmol 1993;111:1210-8.

12 Parks DJ, Abrams DA, Sarfaraji FA, et al. Comparison of topical ciprofloxacin to conventional antibiotic therapy in the treatment of ulcerative keratitis. Am $f$ Ophthalmol 1993;115:471-7.

13 O'Brien TP, Maguire MG, Fink NE, et al. Efficacy of ofloxacin vs cefazolin and tobramycin in the therapy for bacterial keratitis. Arch Ophthalmol 1995;113:1257-65.

14 Hyndiuk RA, Eiferman RA, Caldwell DR, et al. Comparison of ciprofloxacin ophthalmic solution $0.3 \%$ to fortified tobramycin-cefazolin in treating bacterial corneal ulcers. Ophthalmology 1996;103:1854-63.

15 The Ofloxacin Study Group. Ofloxacin monotherapy for the primary treatment of microbial keratitis: a double-masked, randomized, controlled trial with conventional dual therapy. Ophthalmology 1997;104:1902-9.

16 Osborn E, Baum JL, Ernst C, et al. The stability of ten antibiotics in artificial tear solutions. Am $\mathcal{f}$ Ophthalmol 1976;82:775-80

17 Knauf HP,Silveny R, Southern PM Jr, et al. Susceptibility of corneal and conjunctival pathogens to ciprofloxacin. Cornea 1996;15:66-71.

18 Bower KS, Kowalski RP, Gordon YJ. Fluoroquinolones in the treatment of bacterial keratitis. Am f Ophthalmol 1996; 121:712-5.

19 Kunimoto DY, Sharma S, Garg P, et al. In vitro susceptibility of bacterial keratitis pathogens to ciprofloxacin emerging resistance. Ophthalmology 1999;106:80-5.

$20 \mathrm{McG}$ arvey WC, Singh D, Trevino SG. Partial Achilles tendon ruptures associated with fluoroquinolone antibiotics: a case report and literature review. Foot and Ankle Int 1996;17:496-8. 